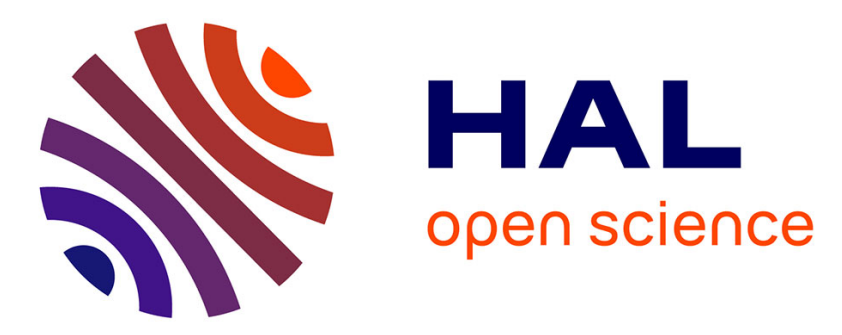

\title{
Investigation of porous anodic alumina templates formed by anodization of single-crystal aluminum substrates
}

\author{
Leandro Sacco, Ileana Florea, Marc Châtelet, Costel-Sorin Cojocaru
}

\section{To cite this version:}

Leandro Sacco, Ileana Florea, Marc Châtelet, Costel-Sorin Cojocaru. Investigation of porous anodic alumina templates formed by anodization of single-crystal aluminum substrates. Thin Solid Films, 2018, 660, pp.213-220. 10.1016/j.tsf.2018.06.015 . hal-02325461

\section{HAL Id: hal-02325461 \\ https://hal.science/hal-02325461}

Submitted on 24 Oct 2019

HAL is a multi-disciplinary open access archive for the deposit and dissemination of scientific research documents, whether they are published or not. The documents may come from teaching and research institutions in France or abroad, or from public or private research centers.
L'archive ouverte pluridisciplinaire HAL, est destinée au dépôt et à la diffusion de documents scientifiques de niveau recherche, publiés ou non, émanant des établissements d'enseignement et de recherche français ou étrangers, des laboratoires publics ou privés. 


\title{
Investigation of porous anodic alumina templates formed by anodization of
}

\section{single-crystal aluminum substrates}

\author{
Leandro Sacco, Ileana Florea, Marc Châtelet, Costel-Sorin Cojocaru \\ LPICM, Ecole Polytechnique, CNRS, Université Paris Saclay, 91128 Palaiseau, France
}

A B S TR A C T

Ordered porous anodic alumina (PAA) templates are of great interest as they facilitate the future development of nanodevices. The present study focuses on the impact of substrates with different crystallographic orientations on the template's pore structure. Characteristics such as pore diameter, interpore distance, pore regularity, porosity, and circularity are calculated as a function of the anodization potential for three different Al crystal orientations. The presented experiments reveal that the different crystallographic orientations mainly impact the pore ordering, while other structural parameters, such as the pore diameter and interpore distance, are not significantly affected.

\author{
Keywords: \\ Porous anodic alumina \\ Single-crystal aluminum \\ Nanostructures \\ Pore ordering
}

\section{Introduction}

During the last decades, porous anodic alumina (PAA) templates have been proposed for a large variety of nanotechnological applications due to their ordered honeycomb structure which can be used for the fabrication of other nanostructures [1-7]. Such nano-templates are highly versatile since their fabrication process enables precise control of the pore-cell size by adjusting the parameters involved in the anodization process [ $8-10]$. The regularity of the pore arrangement, in terms of their pore ordering and pore size uniformity, is an essential requirement for its application as templates and takes even more relevance in certain applications such as interferometric biosensors [11] and photonic crystals [12]. In order to fabricate large areas PAA templates with perfect hexagonal arrays, different approaches have been implemented. However, such procedures generally involve lithography steps [13, 14], plasma treatments [15, 16], mask fabrications [17, 18], atomic force microscopy implantation [19, 20], or a combination of these methods [21]. Most of these procedures carry a high cost, are time-consuming and presenting serious scaling limitations.

The simplest and most straightforward procedure to obtain self-organized PAA arrays is by using the two-step anodization procedure [22]. The methodology consists in the formation of a first PAA layer, followed by its subsequent removal and further re-anodization of the remaining aluminum. The dissolution of the vertically oriented cylindrical pores, created during a first anodization step, leads to a hexagonally ordered patterned aluminum surface consisting of nano-concave structure that serves as nucleation sites for the second anodization step. The pores generated during the second anodization step occurs at the bottom of each concave nucleation site due to a favored dielectric breakdown characterized by a relatively thin native oxide. Here the electric field is the highest and the resistance is the lowest [23, 24]. The chosen anodization conditions are critical parameters for achieving a PAA surface with a perfect order [25]. Nevertheless, the local crystal orientation of the Al substrate, the impurity concentrations, as well as the aluminum grains sizes [26, 27] also play a role and must be considered during the fabrication process. To this account, the "perfect" hexagonal pattern for the nanopores could only be achieved in domains dependent on these grains size that typically have a lateral distension from $100 \mathrm{~nm}$ up to several $\mu \mathrm{m}$ [28]. In order to get a better understanding of the pore's formation and the regularity of the pores that can be obtained, previous studies have focused on the influence of the aluminum substrate over the porous structure of the as-fabricated PAA templates $[29,30]$. In particular, studies regarding the influence of the aluminum substrate's crystal orientation have been realized [31-34], but these studies were mainly devoted to the investigation of the single crystal aluminum orientation's impact upon the periodicity of the pores without considering their morphological characteristics.

The main objective of the present work is to fill the lack of information regarding the pore's morphological characteristics, such as the pore diameter and the interpore distance, when the anodization process is carried out on single crystal aluminum substrates. Herein, we perform a detailed characterization of the pore features of PAA layers formed via two-step anodization process in a $0.3 \mathrm{M}$ oxalic acid solution, for three different single-crystal orientations. The main structural parameters are analyzed for PAA layers obtained in a voltage range from 20 to $60 \mathrm{~V}$. These parameters are: the pore diameter, the interpore

\footnotetext{
* Corresponding author

E-mail address: leandro.sacco@polytechnique.edu (L. Sacco).
} 

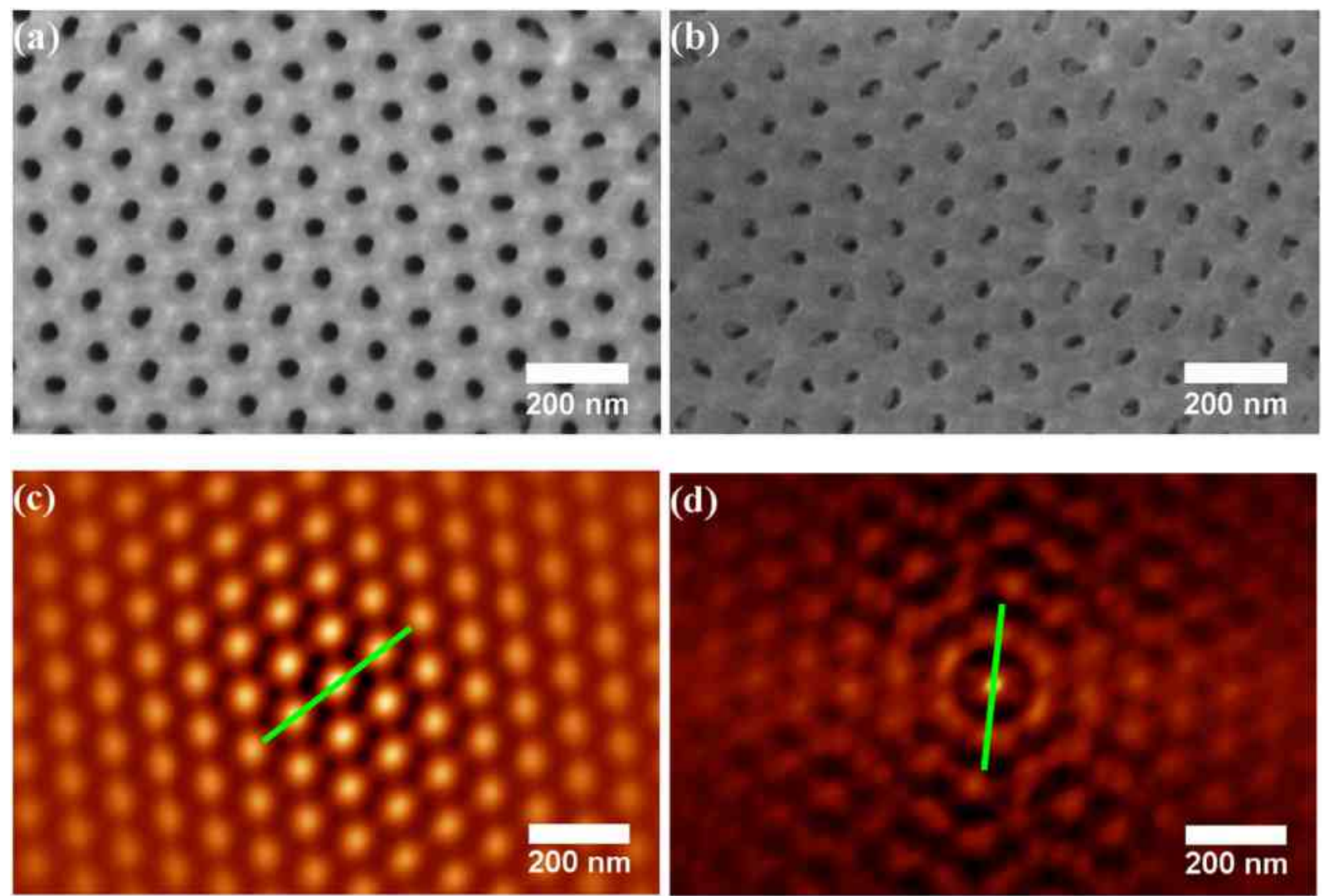

(e)

(f)
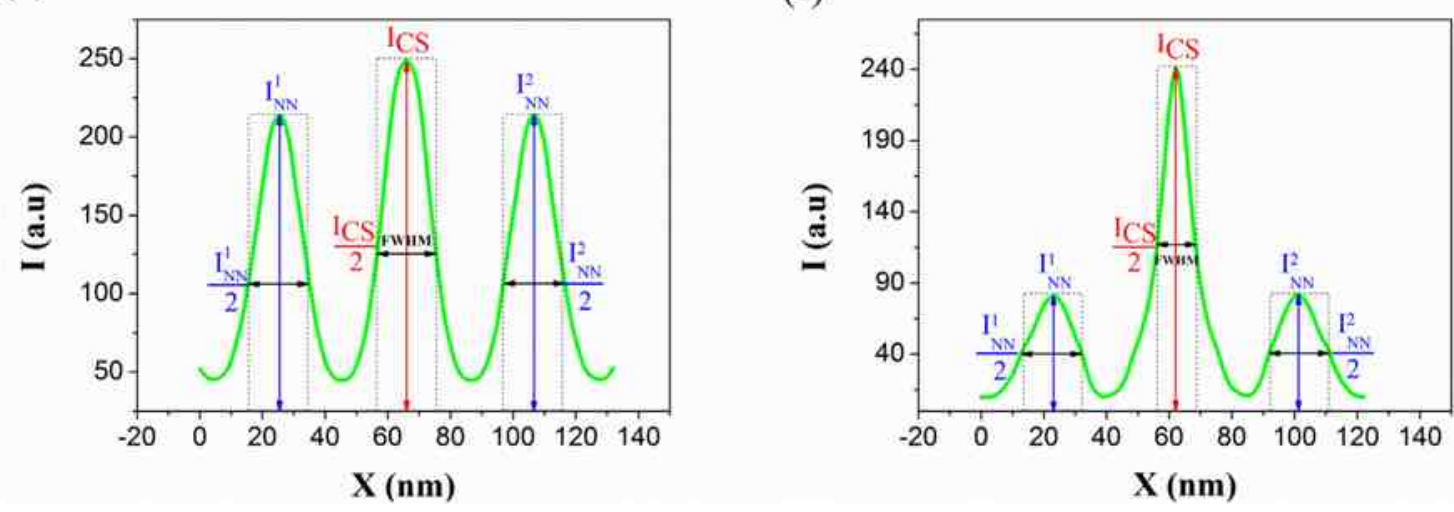

Fig. 1. Calculation of NSOP value to quantitatively determine the pore ordering degree of the PAA layers.

FE-SEM micrographs of PAA template presenting two different pore regularity (a) highly ordered and (b) poorly ordered. (c) and (d) the corresponding two-dimensional SCI, with a line to calculate their intensity pro file (e) and (f).

distance, the porosity, circularity and regularity degree of the pores. The present work highlights a complete correlation between the aluminum substrate crystal orientation and the resulting pore characteristics in terms of their regularity, position and shape.

\section{Materials and methods}

\subsection{PAA fabrication}

High-purity (99.999\%) aluminum monocrystals (Diameter: $12 \mathrm{~mm}$, Length: $50 \mathrm{~mm}$ ) purchased from Goodfellows, with three different orientations (100), (110) and (111) were used as substrates for the PAA templates preparation. Firstly, the aluminum substrates were cleaned with acetone followed by isopropanol, both in an ultra-sonic bath during $15 \mathrm{~min}$. Subsequently, the substrates were electropolished for
6 min while applying $20 \mathrm{~V}$ potential in a bath containing a mixture solution of $1: 4 \mathrm{HClO}_{4}: \mathrm{C}_{2} \mathrm{H}_{5} \mathrm{OH}$ at $17.5^{\circ} \mathrm{C}$

The two-step anodization process was performed in a two-electrode system. A graphite electrode was used as a cathode. The distance between the two electrodes was kept at $2 \mathrm{~cm}$. In order to maintain the electrolyte's temperature constant, all anodization procedures were carried out in a double-walled electrochemical cell combined with a refrigerated circulating system. The anodization processes was carried out in a $0.3 \mathrm{M}$ oxalic acid solution at $17.5^{\circ} \mathrm{C}$ applying a potential in the range of $20 \mathrm{~V}$ to $60 \mathrm{~V}$. The first anodization steps were executed for a duration time of $2 \mathrm{~h}$. Subsequently, the as-formed PAA was selectively removed in a solution containing $0.2 \mathrm{M} \mathrm{CrO}_{3}$ and $0.6 \mathrm{M} \mathrm{H}_{3} \mathrm{PO}_{4}$ at $60{ }^{\circ} \mathrm{C}$. Then a second anodization step was executed under the same conditions as the first one. 


\subsection{Sample characterization}

PAA surface imaging was carried out with a field-emission scanning electron microscope (FE-SEM, HITACHI S4800) after drying the samples at room temperature. In order to avoid any charge effects the acceleration voltage was set at $10 \mathrm{kV}$, under a vacuum level of $5 \times 10^{-4} \mathrm{~Pa}$. The main pore characteristics of the PAA templates, such as the pore area $(A)$, the pore diameter $\left(D_{p}\right)$ and perimeter of the pores $(P)$ are derived directly from the SEM micrographs using image treatment through the open-source "ImageJ" software [35]. Accessing the pore's characteristics enables deducing global characteristics such as the porosity $(\alpha)$, the interpore distance $\left(D_{1}\right)$ and the circularity of the pores $(C)$. The porosity is defined as the ratio between the summed area of each pore $\left(A_{P i}\right)$ with respect to the whole substrate surface area $(A)$, as expressed the following equation:

$\alpha=\frac{\sum_{i} A_{P i}}{A}$

Assuming that every single pore is a perfect circle within a structure presenting hexagonally arranged cells, Eq. (1) can be further developed to provide an expression for the interpore distance in terms of the porosity and the pore diameter:

$D_{l}=D_{P} \cdot \sqrt{\frac{2 \sqrt{3} \alpha}{\pi}}$

A deviation of the pore's shape from an ideal circle can be described by a circularity coefficient (C), defined as:

$C=4 \pi \cdot\left(\frac{A_{P}}{P^{2}}\right)$

The circularity value of 1.0 indicates that the pore's shape is ideally circular, while values close to 0.0 indicate the presence of a deformed irregular shape that would correspond more to a polygon.

In order to determine a quantitative value of the regularity of the pores, a self-correlation function (SCF) was applied to the PAA's top surface images using the freeware software "WSxM4.0" [36], through a procedure previously introduced by Mínguez-Bacho et al. [37]. This approach takes into consideration large surface areas and self-correlated images $(\mathrm{SCl})$, presenting an alternative procedure to Fast Fourier Transform (FFT) and Pair Distribution Function analyses. The resulting self-correlated image is a mathematical image in real space that gives information of how an image correlates with itself under conditions where the initial image is displayed in all possible directions.

The quantitative value of the pores periodicity can be extracted from the Normalized Spatial Order Parameter (NSOP) which is given by Eq. (4).

$N S O P=\frac{1}{6} \cdot \frac{\sum_{N N=1}^{6} \frac{I_{N N}}{F W H M_{N N}}}{\frac{I_{C S}}{F W H M C S}}$

The intensity (I) and the full width at half maximum (FWHM) of both central spot (CS) and nearest neighbor (NN) of three crossing center profiles are measured on the self-correlated images (SCl). These profiles that link the opposite vertex of a hexagon formed by the relative maxima are measured every $60^{\circ}$. Fig. 1 shows the steps involved to extract the coefficients I NN, FWHM ${ }_{\mathrm{NN}}, \mathrm{I}_{\mathrm{CS}}$, and $\mathrm{FWHM}_{\mathrm{CS}}$ As an example, Fig. 1(a) and (b) illustrates the SEM images corresponding to PAA layers presenting two different pore order degree, Fig. 1(a) PAA with a hexagonal pattern, and Fig. 1(b) PAA with several defects. Depending on the regularity of the pores the corresponding SCls are obtained. As it can be observed, Fig. 1(c) presents a well-defined hexagonal pattern while Fig. 1(d) SCF leads to a central spot surrounded by a ring, this is associated with poor order pore distributions. In the cross profile, the pore regularity is related with the relative intensity of CS and NN. As shown in Fig. 1(e), when the intensity of the NN of the central spot is close to the intensity of the CS, the NSOP value is close to
1 , which indicates a highly ordered hexagonally array. On the contrary, when the CS is surrounded by a ring, the intensity of the NN differs significantly from the intensity of the CS, reflecting a low pore regularity, as is displayed in Fig. 1(f). This kind of pattern cannot be treated by the Self-correlated approach to extract quantitative information, to analyze such pore distributions more robust and complex techniques are required as Toccafondi et al. have developed [38].

The pores characteristics, such as pore diameter, interpore distance, porosity, circularity and NSOP have been calculated at 10 different spots, in three different samples, for each single crystal orientation.

\section{Results and discussion}

It is well known that anodization times between 12 and $24 \mathrm{~h}$ are required for anodization processes performed at temperatures between 0 and $5{ }^{\circ} \mathrm{C}$ [39], however at relatively high temperatures $\left(15-30^{\circ} \mathrm{C}\right)$ the oxide growth rate increases, approximately exponentially, with temperature leading to a faster self-organization. Consequently, the first anodization time can be meaningfully reduced [40]. In the present work, the impact of the first anodization step's duration on the pore regularity was studied. To this end, different first self-organized times have been used to fabricate PAA templates. Fig. 2 shows SEM images and their corresponding SCl. The shown results belong to single-crystal substrates with (111) orientation, however similar results have been obtained with the other crystals orientations. The NSOP error value in each case is \pm 0.1 . Under the implemented anodization conditions (i.e $0.3 \mathrm{M}$ oxalic acid, $40 \mathrm{~V}, 17.5^{\circ} \mathrm{C}$ ), the presented experiments reveal that a $2 \mathrm{~h}$ first step anodization duration is enough to achieve the best pore ordered distribution.

In order to identify the relation between the anodization potential and the structural parameters of the formed PAA layer, different aluminum substrates have been anodized with potentials ranging from $20 \mathrm{~V}$ up to $60 \mathrm{~V}$ with an increment of $10 \mathrm{~V}$. Fig. 3 shows the typical evolution of the current density as a function of time: first current shutdown, associated with the formation of a highly resistive dielectric layer, occurs. Then current pick-up followscaused by the propagation of individual paths (pores precursors) through the barrier layerleading to the breakdown of the tight barrier film and the creation of the porous structure., Finallyan equilibrium between the dissolution and formation of alumina is established as the pore growth pursues at a steady phase related to a pore straight formation [41], shown by the leveling of the current.

We can observe that for the applied anodization voltages in the range of $30-60 \mathrm{~V}$, the substrates presenting the (100) crystal orientation reach the steady-state phase faster than the substrates presenting the two other crystal orientations. Fig. 3 points out the time where the anodization current is stabilized for each crystal orientation, the beginning of the steady state regime herein is simply to identy because correspond with the maximum current density value. These observations suggest a faster propagation of individual paths (pores precursors) through the dielectric barrier when a (100) crystal orientation is considered. On the other hand, we can assume that for the three crystal orientations there are no statistically significant differences of the current value at the stationary stage for a certain anodization potential Curves shown in Fig. 3 correspond to a first anodization process. Previous reports have established a linear relationship between the pore diameter $\left(D_{P}\right)$ and interpore distance $\left(D_{1}\right)$ of the PAA with the applied potential when a stationary regime is achieved for the pore's growth. Therefore it is generally accepted that $D_{P}$ and $D_{1}$ can be expressed as a function of the anodization voltage as in Eqs. (5) and (6), where $\lambda_{p}$ and $\lambda_{\text {I }}$ are proportionality constants.

$D_{P}=\lambda_{P} * U_{\text {Anod }}$

$D_{I}=\lambda_{I} * U_{\text {Anod }}$

However former studies also confirm that the proportionality 

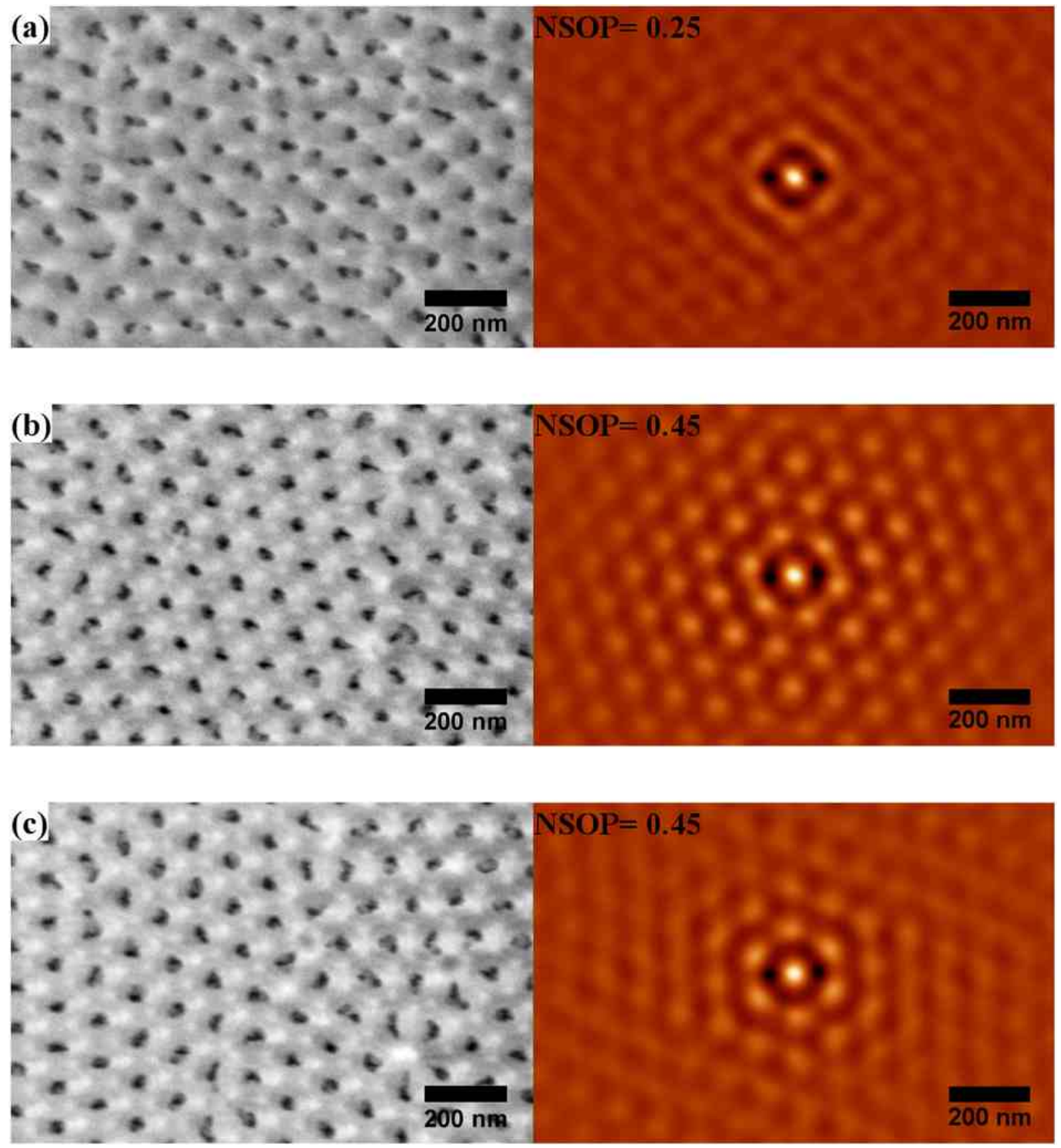

Fig. 2. SEM images and their corresponding self-correlated image (SCl) of PAA oxide layers after the second step of anodization. Di fferent self-organization times during the first anodization step have been imposed: (a) $0.15 \mathrm{~h}$, (b) $2 \mathrm{~h}$ and (c) $4 \mathrm{~h}$.

constants values are directly dependent on the anodization conditions and the substrate characteristics. Thus it has been shown that the $A$ and $\lambda_{\text {I }}$ can vary from $0.47 \mathrm{~nm} / \mathrm{V}$ [42] to $1.38 \mathrm{~nm} / \mathrm{V}$ [43] and from $2.00 \mathrm{~nm} /$ $\mathrm{V}[44]$ to $2.81 \mathrm{~nm} / \mathrm{V}$ [9], respectively.

In our case, as summarized in Table 1 , we calculated $\lambda_{p}$ and $\lambda_{1}$ for the fabricated PAA templates for the three crystal orientation of the substrate. For instance, in Fig. 4 we show SEM top images corresponding to PAA structures fabricated on the three different crystallographic orientations of the aluminum substrates using an anodization voltage of $40 \mathrm{~V}$. Insets show their corresponding SCl that served as support for the calculation of the pore's structure parameters.

The dependence of the measured pore diameter and the interpore distance as a function of the anodization potential, for the three different aluminum substrates orientations, are shown in Fig. 5. Table 1 summarises the results obtained for $\lambda_{P}$ and $\lambda_{\text {I }}$ calculated using a linear fit. The calculation was performed for the PAA structures obtained using anodization potential ranging from $20 \mathrm{~V}$ to $50 \mathrm{~V}$. As it can be depicted from Table 1, higher anodization potentials lead to very disordered pore configuration, consequently, a high dispersion for the pore diameter and interpore distance was calculated. The high current density that is induced under such anodization conditions leads to the formation of disorder porous structures. This result is in agreement with previous works which stated that the optimal anodization potential in such acidic environment corresponds to $40 \mathrm{~V}$ [43-45]. In addition, the results obtained from Table 1 shows that PAA structures, fabricated with the used single-crystal aluminum substrates, have proportionality constants values that overlap in the range: $0.90 \leq \lambda_{p} \leq 1.10$ and $2.35 \leq \lambda_{1} \leq 2.50$. These findings suggest that using substrates 


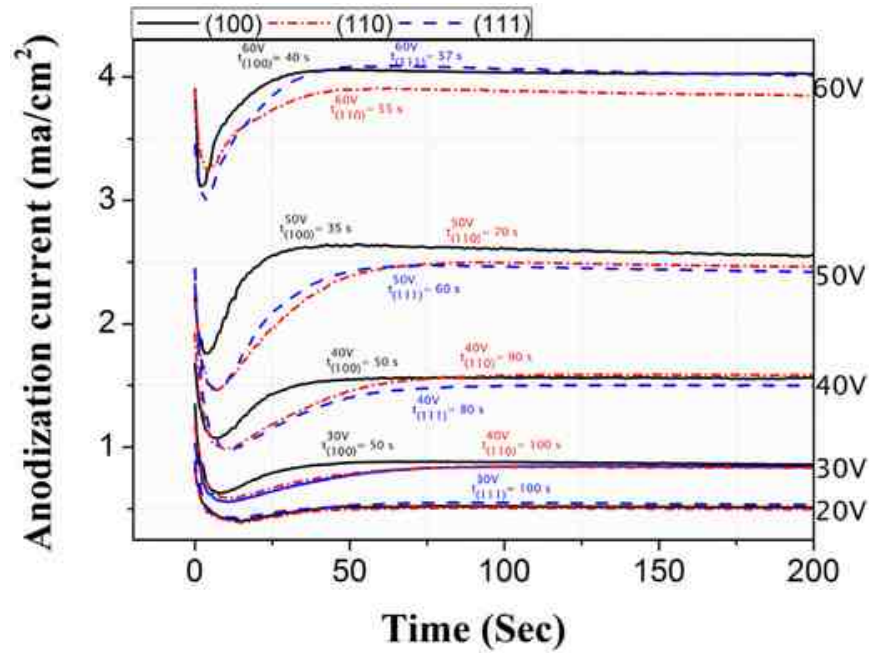

Fig. 3. Current density as a function of time for three different single crystal orientations of the aluminum substrates. The anodization potential has been varied in the range of $20-60 \mathrm{~V}$ with an increment of $10 \mathrm{~V}$. For each aluminum substrate is indicated time value where the anodization current is stabilized for each crystal orientation.

Table 1

Calculated values for $\lambda_{P}$ and $\lambda_{1}$ for the PAA templates fabricated using the anodization process for the aluminum substrates with the three considered single crystal orientations.

\begin{tabular}{lll}
\hline Aluminum crystal orientation & $\lambda_{\mathrm{P}}[\mathrm{nm} / \mathrm{V}]$ & $\lambda_{\mathrm{I}}[\mathrm{nm} / \mathrm{V}]$ \\
\hline$[100]$ & $1.10 \pm 0.10$ & $2.50 \pm 0.25$ \\
{$[110]$} & $0.90 \pm 0.10$ & $2.35 \pm 0.20$ \\
{$[111]$} & $1.05 \pm 0.10$ & $2.45 \pm 0.25$ \\
\hline
\end{tabular}

presenting different single-crystallographic orientations does not significantly affects the pore diameter and interpore distance.

In order to improve the characterization of the PAA structures, detailed calculation the pores regularity, porosity, and circularity has been performed. Fig. 6(a) clearly indicates that for all the fabricated porous structures the optimal pore ordering is obtained when an anodized potential of $40 \mathrm{~V}$ is used. The self-correlated technique is a fast and powerful tool to establish the pore regularity for PAA with relatively ordered structure. This is the case of the PAA fabricated with potentials ranged from $30 \mathrm{~V}$ to $50 \mathrm{~V}$ where the respective $\mathrm{SCl}$ present hexagonal patterns. However, when the anodization potentials are $20 \mathrm{~V}$ or $60 \mathrm{~V}$, the resulting $\mathrm{SCl}$ are characterized by a ring surrounding the $\mathrm{CS}$, reflecting a poorly order structure, leading to NSOP values that are close to zero. In order to establish the order degree of arrays that differ strongly from an ideal pattern, more robust surface processing tools are required, as Toccafondi et al. have previously reported [38]. Therefore, we can establish that for PAA layers fabricated within the potential range $30-50 \mathrm{~V}$, the resulting structures are the most regular for aluminum substrates with (100) crystal orientations, followed by aluminum substrate with (111) orientation and subsequently by (110) crystal orientation. Such results are in perfect agreement with previous works which verified that pore ordering depends on the crystallographic orientation of the aluminum substrates [31, 32, 45]. In particular, Beck and Bretzler have shown that for aluminum anodized in sulfuric acid solution at a potential of $25 \mathrm{~V}$, also follows the subsequent rank of ordering quality as a function of the single crystal orientation $(100)>(111)>(110)$. Our results are complementary to these previous reports both by providing significant data concerning anodization in an oxalic acid solution and also by presenting a more extended study covering the range of anodization potentials from $30 \mathrm{~V}$ to $50 \mathrm{~V}$.

The porosity value as a function of the anodization voltage was also
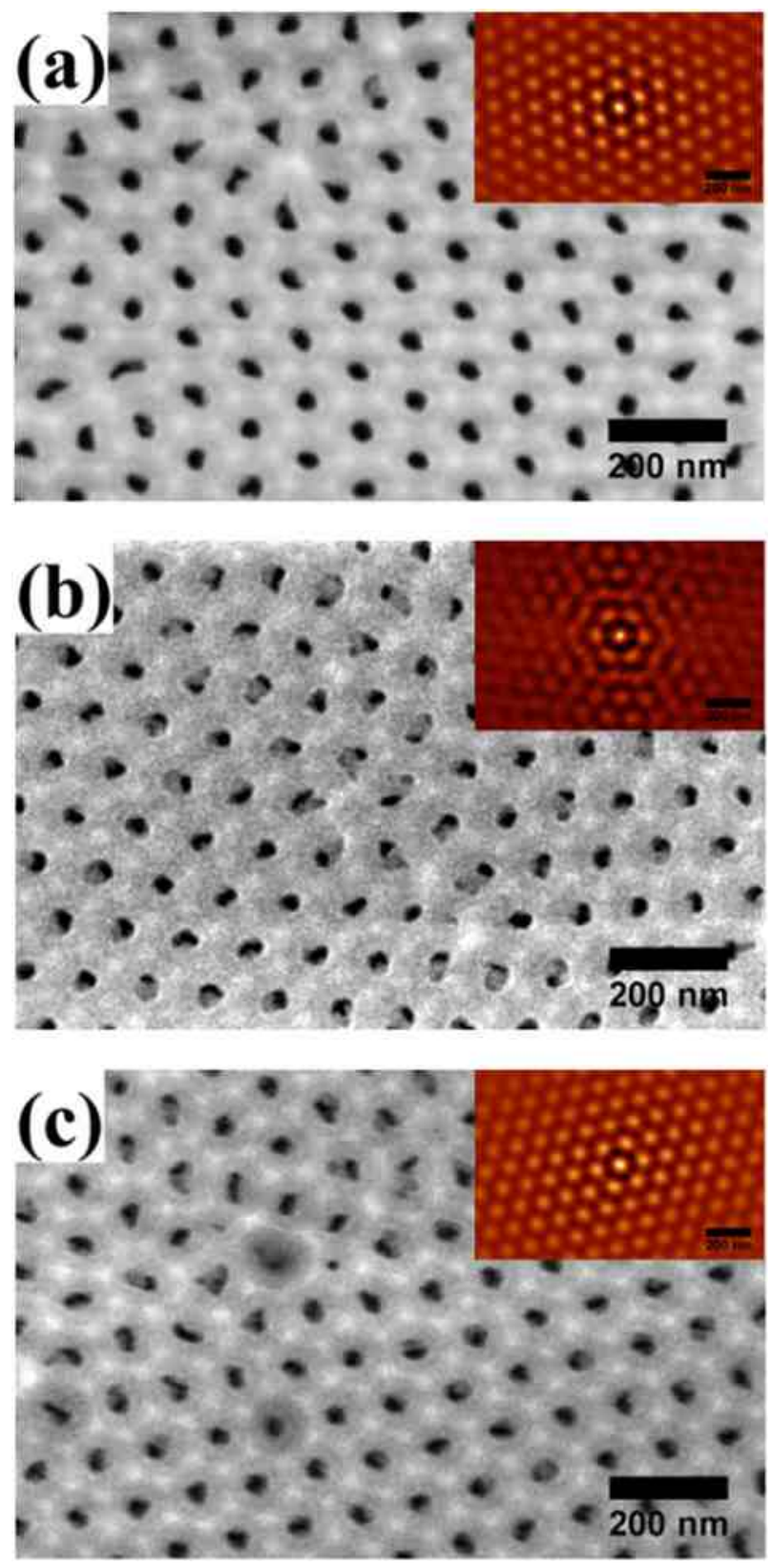

Fig. 4. SEM top images of the PAA structures fabricated in $0.3 \mathrm{M}$ oxalic acid using an anodization potential of $40 \mathrm{~V}$ for (a) (100) aluminum single-crystal orientation, (b) (110) aluminum single-crystal orientation (c) (111) aluminum single-crystal orientation. With ininset the corresponding self-correlated image (SCl).

estimated and is plotted in Fig. 6(b). It is interesting to highlight that for all the three single-crystal aluminum substrates, the porosity reaches the maximum value when the samples are anodized at $40 \mathrm{~V}$, which also corresponds to the best pores ordering distribution. In all cases, such values are around $12 \pm 2 \%$. It has been proven by Nielsch et al. [8] that the best pore hexagonal arrangement is correlated with approximately $10 \%$ porosity. Nevertheless, such a rule is valid for mild anodization processes. To the contrary, for hard anodization processes, the porosity in the best self-organize regime is $3.3-3.4 \%$. The results obtained in the present work, make us conclude that the anodization conditions correspond to a mild process.

Finally, the last feature examined is the pores circularity as shown in Fig. 6(c). As it can be depicted from the figure for all cases, the most circular pores are obtained when a potential of $50 \mathrm{~V}$ is used for the 
(a)

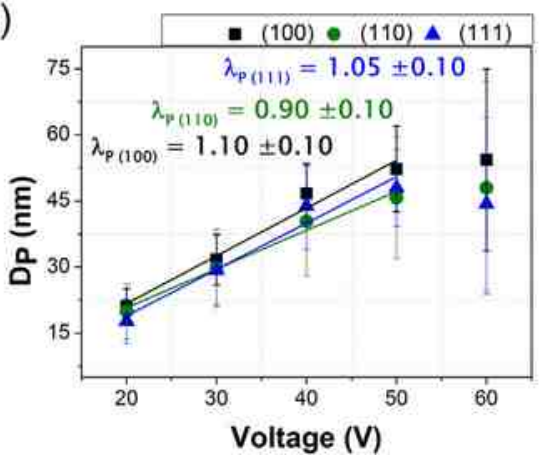

(b)

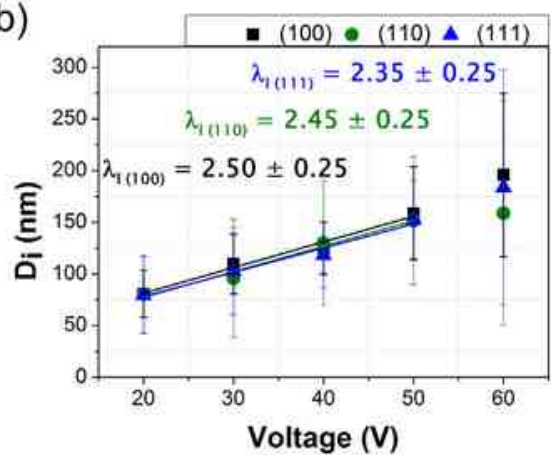

Fig. 5. Variation of the pore diameter (a) and the interpore distance (b) for PAA layers fabricated from three different single-crystal aluminum substrates with the (100), (110) and (111) crystallographic orientation. Linear fitting are performed to extract $\lambda_{P}$ and $\lambda_{1}$ proportionality constants in the anodization potential ranged from $20 \mathrm{~V}$ to $50 \mathrm{~V}$. anodization step. Such result is quite surprising with respect to previous ones and gives a clear identification of the best pore distribution that can be obtained using an anodization potential of $40 \mathrm{~V}$. At this stage of analysis, the self-correlated method does not strictly calculate the pore ordering in terms of their relative position. The degree of spatial ordering of the PAA structures can be calculated quantitatively by using the radial distribution function (RDF) approach [32]. However, this method does not consider the pore shape. On the other hand, the selfcorrelated approach takes into account the shape of the pore, as well as their relative position, providing quantitative information of the pore regularity. Therefore similar pores lead to a more regular pore distribution. In order to corroborate these assumptions, a pore widening process has been performed on a PAA structure. It is well known that such process etches in an isotropic manner, making the PAA structure more circular.

Fig. 7 shows a comparison of two PAA structures fabricated under the same conditions using a single-crystal aluminum substrate with a (100) crystallographic orientation. The PAA structure without widening is presented in Fig. $7(\mathrm{a})$ and the structure submitted to the pore widening process in a $0.3 \mathrm{M} \mathrm{H}_{3} \mathrm{PO}_{4}$ solution during 25 min at $30^{\circ} \mathrm{C}$, is presented in Fig. $7(\mathrm{~b})$. One can easily observe that after the pore widening treatment, the pores circularity increases in about $5 \%$, leading to an increased pore regularity of about $10 \%$, although the position of the pores remains the same. However, given the errors involved, it should be pointed out that the difference is not statistically significant to conclude the pore wedding effect on the pore regularity. Longer pore wedding times should be executed in order to obtain more conclusive results.

Nevertheless, comparing these findings with those plotted in Fig. 5(a) and Fig. 5(c) we can conclude that in general, the positions of the pores are more disorder when the substrates are anodized at $50 \mathrm{~V}$ than at $40 \mathrm{~V}$ but by contrast, at $50 \mathrm{~V}$ the obtained pores possess a more circular shape than those obtained through anodization at $40 \mathrm{~V}$. This can be attributed to a higher local temperature through Joule effect, at the electrolyte/oxide interface when the applied potential is $50 \mathrm{~V}$, which leads to a more effective field-assisted isotropic dissolution of aluminum oxide.

\section{Conclusions}

The present work corroborates several significant pore characteristics affected by the different crystallographic orientation ((100), (110) and (111)) of the aluminum substrates when an anodization process is carried out in oxalic acid with anodization voltages from $30 \mathrm{~V}$ to $50 \mathrm{~V}$. We have shown that the substrate crystallographic orientation impacts directly on the pores regularity but not in a meaningful way over their diameter and interpore distance. As previously reported, the rank of quality in terms of ordering degree depending on the crystallographic orientation, for all the anodization potentials, verifies that the best ordering is obtained when (100) aluminum substrates are used for the synthesis, followed by those with a (111) crystallographic orientation. The present work also points out that the most disorder structures are obtained for aluminum substrates with the (110) orientation. The highest porosity values are correlated with the most regulars pore's distributions fabricated at $40 \mathrm{~V}$ in accordance with the $10 \%$ rule. In contrast, the more circular pores are obtained with anodization potentials of $50 \mathrm{~V}$. This can be attributed to the enhancement of a fieldassisted isotropic dissolution of aluminum oxide occurring during anodizing in the acidic electrolyte induced by higher local temperatures at the oxide/electrolyte interface. First studies have been performed to prove that isotropic pore widening processes can lead to an improvement of the pore regularity, consequence of a more homogeneous pore distribution in terms of their shape. However, further studies must be executed in this direction to obtain conclusive results.

\section{Acknowledgments}

L.S gratefully acknowledges financial support from the Chaire de Recherche "André Citroen' (PSA AGM) at the Ecole Polytechnique. The authors would like to thank Dr. Fernando Diaz for proofreading this (a)

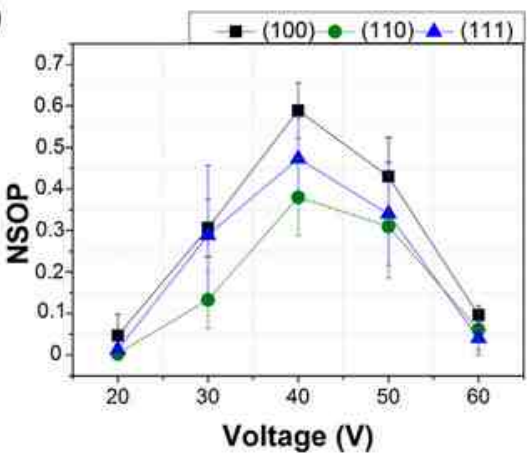

(b)

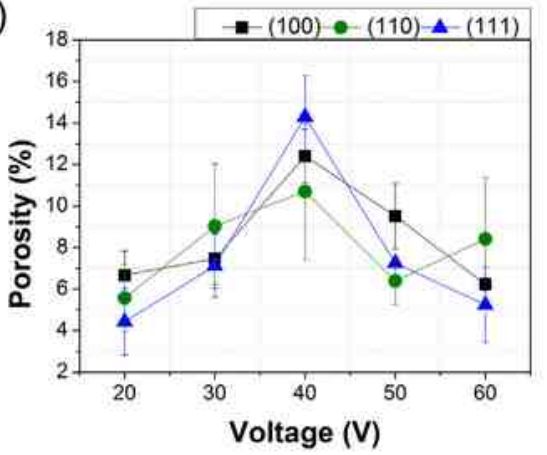

(c)

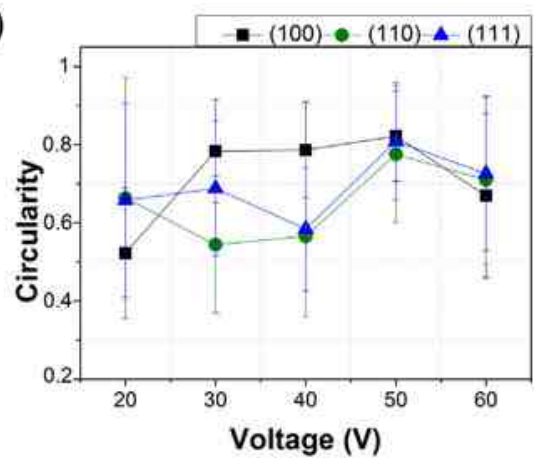

Fig. 6. Pore characteristics as a function of anodization voltage using substrates with different crystallographic orientations. (a) Pore ordering, (b) Porosity and (c) Circularity. 

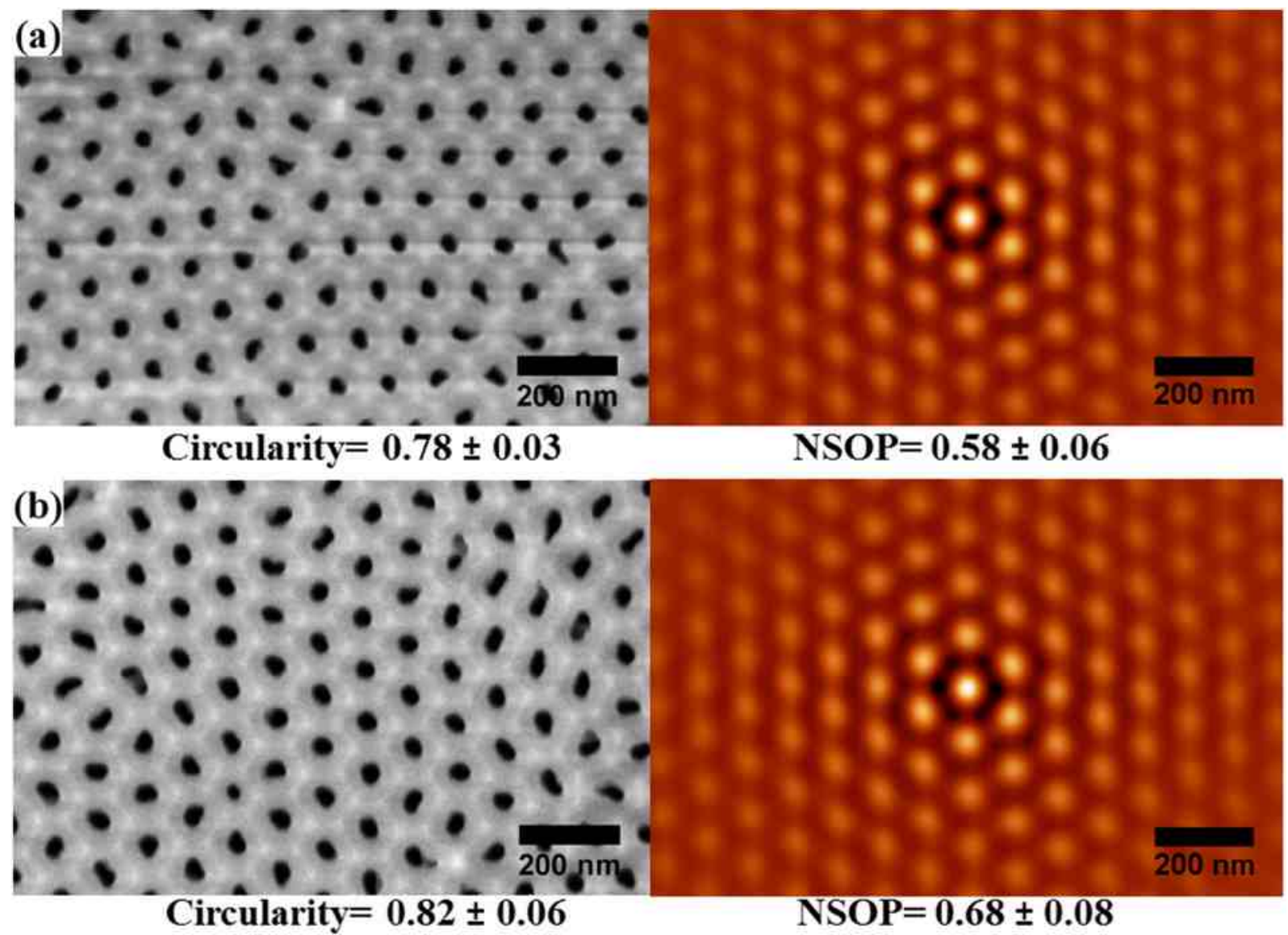

Fig. 7. Comparison of two PAA structures fabricated under the same conditions using a single-crystal aluminum substrate with a (100) crystallographic orientation with their corresponding self-correlated image. (a) PAA obtained by two-step anodization process and (b) pore widening applied after PAA fabrication.

paper.

References

[1] G.D. Sulka, A. Brzózka, L. Zaraska, M. Jaskuła, Through-hole membranes of nanoporous alumina formed by anodizing in oxalic acid and their applications in fabrication of nanowire arrays, Electrochim. Acta 55 (2010) 4368-4376, http://dx. doi.org/10.1016/j.electacta.2010.01.048.

[2] F. Thissandier, L. Dupré, P. Gentile, T. Brousse, G. Bidan, D. Buttard, S. Sadki, Ultradense and highly doped SiNWs for micro-supercapacitors electrodes, Electrochim. Acta 117 (2014) 159-163, http://dx.doi.org/10.1016/j.electacta.2013.11.097.

[3] J. Kim, D.H. Lee, J.H. Kim, S.H. Choi, Graphene-assisted chemical etching of silicon using anodic aluminum oxides as patterning templates, ACS Appl. Mater. Interfaces 7 (2015) 24242-24246, http://dx.doi.org/10.1021/acsami.5b07773.

[4] M.S. Sander, A.L. Prieto, R. Gronsky, T. Sands, A.M. Stacy, Fabrication of highdensity, high aspect ratio, large-area bismuth telluride nanowire arrays by electrodeposition into porous anodic alumina templates, Adv. Mater. 14 (2002) 665-667, http://dx.doi.org/10.1002/1521-4095(20020503)14:9<665::AIDADMA665>3.0.CO;2-B.

[5] T. Wang, Y. Wang, F. Li, C. Xu, D. Zhou, Morphology and magnetic behaviour of an Fe 3 O 4 nanotube array, J. Phys. Condens. Matter. 18 (2006) 10545-10551, http:// dx.doi.org/10.1088/0953-8984/18/47/002.

[6] A. Li, S. Pan, X. Dou, Y. Zhu, X. Huang, Y. Yang, G. Li, L. Zhang, Direct electrodeposition of $\mathrm{ZnO}$ nanotube arrays in anodic alumina membranes, J. Phys. Chem. C 111 (2007) 7288-7291, http://dx.doi.org/10.1021/jp0711242.

[7] K.H. Kim, D. Brunel, A Gohier, L. Sacco, M. Châtelet, C.S. Cojocaru, Cup-stacked carbon nanotube schottky diodes for photovoltaics and photodetectors, Adv. Mater. 26 (2014) 4363-4369, http://dx.doi.org/10.1002/adma.201400775.

[8] W. Chen, J.S. Wu, X.H. Xia, Porous anodic alumina with continuously manipulated pore/cell size, ACS Nano 2 (2008) 959-965, http://dx.doi.org/10.1021/nn700389j.

[9] A.P. Li, F. Müller, A. Birner, K. Nielsch, U. Gösele, Hexagonal pore arrays with a 50-420 nm interpore distance formed by self-organization in anodic alumina, J. Appl. Phys. 84 (1998) 6023-6026, http://dx.doi.org/10.1063/1.368911.

[10] L. Zaraska, G.D. Sulka, M. Jaskula, Anodic alumina membranes with defined pore diameters and thicknesses obtained by adjusting the anodizing duration and pore opening/widening time, J. Solid State Electrochem. 15 (2011) 2427-2436, http:/l dx.doi.org/10.1007/s10008-011-1471-z.

[11] S.D. Alvarez, C.P. Li, C.E. Chiang, I.K. Schuller, M.J. Sailor, A label-free porous alumina interferometric immunosensor, ACS Nano 3 (2009) 3301-3307, http://dx. doi.org/10.1021/nn900825q.

[12] M. Nakao, S. Oku, H. Tanaka, Y. Shibata, A. Yokoo, T. Tamamura, H. Masuda, Fabrication of GaAs hole array as a 2D-photonic crystal and their application to photonic bandgap waveguide, Opt. Quant. Electron. 34 (2002) 183-193, http://dx. doi.org/10.1023/A:1013329631972.

[13] A.P. Li, Polycrystalline and monocrystalline pore arrays with large interpore distance in anodic alumina, Electrochem. Solid-State Lett. 3 (1999) 131, http://dx.doi. org/10.1149/1.1390979

[14] W. Lee, R. Ji, C.A. Ross, U. Gösele, K. Nielsch, Wafer-scale Ni imprint stamps for porous alumina membranes based on interference lithography, Small 2 (2006) 978-982, http://dx.doi.org/10.1002/smll.200600100.

[15] H. Masuda, Y. Matsui, M. Yotsuya, F. Matsumoto, K. Nishio, Fabrication of highly ordered anodic porous alumina using self-organized polystyrene particle array, Chem. Lett. 33 (2004) 584-585, http://dx.doi.org/10.1246/cl.2004.584.

[16] H. Oshima, H. Kikuchi, H. Nakao, K.I. Itoh, T. Kamimura, T. Morikawa, K. Matsumoto, T. Umada, H. Tamura, K. Nishio, H. Masuda, Detecting dynamic signals of ideally ordered nanohole patterned disk media fabricated using nanoimprint lithography, Appl. Phys. Lett. 91 (2007) 10-13, http://dx.doi.org/10. 1063/1.2757118.

[17] H. Masuda, H. Asoh, M. Watanabe, K. Nishio, M. Nakao, T. Tamamura, Square and triangular nanohole array architectures in anodic alumina, Adv. Mater. 13 (2001) 189-192, http://dx.doi.org/10.1002/1521-4095(200102)13:3<189::AIDADMA189>3.0.CO;2-Z.

[18] H. Masuda, H. Yamada, M. Satoh, H. Asoh, M. Nakao, T. Tamamura, Highly ordered nanochannel-array architecture in anodic alumina, Appl. Phys. Lett. 71 (1997) 2770-2772, http://dx.doi.org/10.1063/1.120128.

[19] S. Shingubara, Y. Murakami, K. Morimoto, T. Takahagi, Formation of aluminum nanodot array by combination of nanoindentation and anodic oxidation of aluminum, Surf. Sci. 532-535 (2003) 317-323, http://dx.doi.org/10.1016/S00396028(03)00433-3.

[20] H. Masuda, K. Kanezawa, K. Nishio, Fabrication of ideally ordered nanohole arrays in anodic porous alumina based on nanoindentation using scanning probe microscope, Chem. Lett. 31 (2002) 1218-1219, http://dx.doi.org/10.1246/cl.2002.1218.

[21] Z. Sun, H.K. Kim, Growth of ordered, single-domain, alumina nanopore arrays with holographically patterned aluminum films, Appl. Phys. Lett. 81 (2002) 3458-3460, http://dx.doi.org/10.1063/1.1517719.

[22] H. Masuda, K. Fukuda, Ordered metal nanohole array made by 2-step replication of honeycomb structures of anodic alumina, Science 268 (1995) 1466-1468, http:// 
dx.doi.org/10.1126/science.268.5216.1466.

[23] F. Li, L. Zhang, R. Metzger, On the growth of highly ordered pores in anodized aluminum oxide, Chem. Mater. 10 (1998) 2470-2480, http://dx.doi.org/10.1021/ cm980163a.

[24] G. Thompson, Porous anodic alumina: fabrication, characterization and applications, Thin Solid Films 297 (1997) 192-201, http://dx.doi.org/10.1016/S00406090(96)09440-0.

[25] C.S. Chi, J.H. Lee, I. Kim, H.J. Oh, Effects of microstructure of aluminum substrate on ordered nanopore arrays in anodic alumina, J. Mater. Sci. Technol. 31 (2015) 751-758, http://dx.doi.org/10.1016/j.jmst.2014.09.019.

[26] F. Rashidi, T. Masuda, H. Asoh, S. Ono, Metallographic effects of pure aluminum on properties of nanoporous anodic alumina (NPAA), Surf. Interface Anal. 45 (2013) 1490-1496, http://dx.doi.org/10.1002/sia.5285.

[27] L. Zaraska, E. Kurowska, G.D. Sulka, I. Senyk, M. Jaskula, The effect of anode surface area on nanoporous oxide formation during anodizing of low purity aluminum (AA1050 alloy), J. Solid State Electrochem. 18 (2014) 361-368, http://dx. doi.org/10.1007/s10008-013-2215-z.

[28] G. Beck, K. Petrikowski, Influence of the microstructure of the aluminum substrate on the regularity of the nanopore arrangement in an alumina layer formed by anodic oxidation, Surf. Coatings Technol. 202 (2008) 5084-5091, http://dx.doi. org/10.1016/j.surfcoat.2008.04.089.

[29] S.Y. Cho, J.W. Kim, S.D. Bu, Effects of impurities on phase transition changes according to heat treatment of porous anodic alumina fabricated in oxalic acid and phosphoric acid electrolytes, J. Korean Phys. Soc. 66 (2015) 1394-1400, http://dx. doi.org/10.3938/jkps.66.1394.

[30] A.M. Abd-Elnaiem, A.M. Mebed, A. Gaber, M.A. Abdel-Rahim, Tailoring the porous nanostructure of porous anodic alumina membrane with the impurity control, $\mathrm{J}$. Alloys Compd. 659 (2016) 270-278, http://dx.doi.org/10.1016/j.jallcom.2015.11. 069.

[31] I.V. Roslyakov, D.S. Koshkodaev, A.A. Eliseev, D. Hermida-Merino, A.V. Petukhov, K.S. Napolskii, Crystallography-induced correlations in pore ordering of anodic alumina films, J. Phys. Chem. C 120 (2016) 19698-19704, http://dx.doi.org/10. 1021/acs.jpcc.6b05268.

[32] C.K.Y. Ng, A.H.W. Ngan, Precise control of nanohoneycomb ordering over anodic aluminum oxide of square centimeter areas, Chem. Mater. 23 (2011) 5264-5268, http://dx.doi.org/10.1021/cm202461z.

[33] G. Beck, R. Bretzler, Regularity of nanopores in anodic alumina formed on or ientated aluminium single-crystals, Mater. Chem. Phys. 128 (2011) 383-387, http://dx.doi.org/10.1016/j.matchemphys.2011.03.022.
[34] K.S. Napolskii, I.V. Roslyakov, A.Y. Romanchuk, O.O. Kapitanova, A.S. Mankevich, V.A. Lebedev, A.A. Eliseev, Origin of long-range orientational pore ordering in anodic films on aluminium, J. Mater. Chem. 22 (2012) 11922, http://dx.doi.org/ 10.1039/c2jm31710a.

[35] C.A. Schneider, W.S. Rasband, K.W. Eliceiri, NIH Image to ImageJ: 25 years of image analysis, Nat. Methods 9 (2012) 671-675, http://dx.doi.org/10.1038/nmeth. 2089.

[36] I. Horcas, R. Fernández, J.M. Gómez-Rodríguez, J. Colchero, J. Gómez-Herrero, A.M. Baro, WSXM: A software for scanning probe microscopy and a tool for nanotechnology, Rev. Sci. Instrum. 78 (2007), http://dx.doi.org/10.1063/1.2432410.

[37] I. Mínguez-Bacho, S. Rodríguez-López, A. Asenjo, M. Vázquez, M. Hernández-Vélez, Self-correlation function for determination of geometrical parameters in nanoporous anodic alumina films, Appl. Phys. A Mater. Sci. Process. 106 (2012) 105-112, http://dx.doi.org/10.1007/s00339-011-6569-8.

[38] C. Toccafondi, W.J. Stępniowski, M. Leoncini, M. Salerno, Advanced morphological analysis of patterns of thin anodic porous alumina, Mater. Charact. 94 (2014) 26-36, http://dx.doi.org/10.1016/j.matchar.2014.05.003.

[39] K. Nielsch, J. Choi, K. Schwirn, R.B. Wehrspohn, U. Gösele, Self-ordering regimes of porous alumina: the 10 porosity rule, Nano Lett. 2 (2002) 677-680, http://dx.doi. org/10.1021/nl025537k.

[40] C. Cheng, K.Y. Ng, A.H.W. Ngan, Quantitative characterization of acid concentration and temperature dependent self-ordering conditions of anodic porous alumina AIP Adv. 1 (2011) 42113, http://dx.doi.org/10.1063/1.3655416.

[41] G.D. Sulka, W.J. Stepniowski, Structural features of self-organized nanopore arrays formed by anodization of aluminum in oxalic acid at relatively high temperatures, Electrochim. Acta 54 (2009) 3683-3691, http://dx.doi.org/10.1016/j.electacta. 2009.01.046.

[42] L. Zaraska, G.D. Sulka, J. Szeremeta, M. Jaskuła, Porous anodic alumina formed by anodization of aluminum alloy (AA1050) and high purity aluminum, Electrochim. Acta 55 (2010) 4377-4386, http://dx.doi.org/10.1016/j.electacta.2009.12.054.

[43] A.L. Friedman, D. Brittain, L. Menon, Roles of $\mathrm{pH}$ and acid type in the anodic growth of porous alumina, J. Chem. Phys. 127 (2007), http://dx.doi.org/10.1063/ 1.2790429.

[44] W. Lee, R. Ji, U. Gösele, K. Nielsch, Fast fabrication of long-range ordered porous alumina membranes by hard anodization, Nat. Mater. 5 (2006) 741-747, http://dx. doi.org/10.1038/nmat1717.

[45] C. Cheng, K.Y. Ng, N.R. Aluru, A.H.W. Ngan, Simulation and experiment of substrate aluminum grain orientation dependent self-ordering in anodic porous alumina, J. Appl. Phys. 113 (2013) 204903, http://dx.doi.org/10.1063/1.4807295. 